13. Weaver IC, et al. Epigenetic programming by maternal behavior. Nat Neurosci. 2004; 2004;7(8):847-854

14. Kane MA, et al. Influence on immunoreactive folate-binding proteins of extracellular folate concentration in cultured human cells. J Clin Invest. 1988;81(5):1398-1406

15. Liu M, et al. Structure and regulation of the murine reduced folate carrier gene: identification of four noncoding exons and promoters and regulation of dietary folates. J Biol Chem. 2005;280(7):5588-5597.

16. Rachmandani S, Bhattacharya SK, Cervoni N, Szyf M. DNA methylation is a reversible biological phenomenon. Proc Natl Acad Sci U S A.
1999;96(11):6107-6112.

17. Endres M, et al. DNA methyltransferase contributes to delayed ischemic brain injury. J Neurosci. 2000;20(9):3175-81.

18. Chawla RK, Watson WH, Jones DP. Effect of hypoxia on hepatic DNA methylation and tRNA methyltransferase in rat: similarities to effects of methyldeficient diets. J Cell Biochem. 1996;61(1):72-80.

19. Cerda S, Weitzman SA. Influence of oxygen radical injury on DNA methylation. Mutat Res. 1997;386(2):141-152.

20. Goulian M, Bleile B, Tseng BY. Methotrexateinduced misincorporation of uracil into DNA. Proc
Natl Acad Sci U S A. 1980;77(4):1956-1960.

21. Hagen L, et al. Genomic uracil and human disease. Exp Cell Res. 2006;312(14):2666-672.

22. Shen JC, Rideout WM 3rd, Jones PA. High frequency mutagenesis by a DNA methyltransferase. Cell. 1992;71(7):1073-1080.

23. Kronenberg $G$, et al. Folate deficiency induces neurodegeneration and brain dysfunction in mice lacking uracil DNA glycosylase. J Neurosci. 2008;28(28):7219-7230.

24. Shen $S$, et al. Age-dependent epigenetic control of differentiation inhibitors is critical for remyelination efficiency. Nat Neurosci. 2008;11(9):1024-1034.

\title{
p63 and IRF6: brothers in arms against cleft palate
}

\author{
Amel Gritli-Linde
}

Department of Oral Biochemistry, Institute of Odontology, Sahlgrenska Academy, University of Gothenburg, Göteborg, Sweden.

\begin{abstract}
Cleft lip and cleft palate, which can also occur together as cleft lip and palate, are frequent and debilitating congenital malformations, with complex geneses that have both genetic and environmental factors implicated. Mutations in the genes encoding the p53 homolog p 63 and interferon regulatory factor 6 (IRF6) are major causes of cleft lip and cleft palate, but the molecular and cellular mechanisms underlying this have not been clear. However, in this issue of the JCI, Thomason et al. and Moretti et al. independently show that $\mathrm{p} 63$ and IRF6 operate within a regulatory loop to coordinate epithelial proliferation and differentiation during normal palate development. Disruption of this loop as a result of mutations in $p 63$ or IRF6 causes congenital clefting.
\end{abstract}

The developing organism is a master at using simple paradigms to generate complexity. This is typified by the use, at least during early organogenesis, of the same molecular pathways to drive the development of organs and tissues as diverse as the brain, limbs, teeth, and skin. This, however, has a major disadvantage, in that disruption of a specific molecular pathway can lead to concomitant dysfunction of multiple organs, a condition clinically classified as a syndrome.

In humans, mutations in the gene encoding the transcription factor p63, a p53 homolog, cause several autosomal dominant ectodermal dysplasia syndromes (i.e., syndromes deriving from abnormalities in ectodermal structures, such as the hair, teeth, nails, sweat glands, craniofacial structures, and digits), including ectrodactylyectodermal dysplasia-clefting (EEC) and ankyloblepharon-ectodermal dysplasiaclefting (AEC) syndromes. These are both

Conflict of interest: The author has declared that no conflict of interest exists.

Citation for this article: JClin Invest. 2010; 120(5):1386-1389. doi:10.1172/JCI42821. characterized by cleft lip and/or cleft palate $(\mathrm{CL} / \mathrm{CP})$ and defects in ectoderm-derived tissues, such as the epidermis, hair, teeth, and glands (1). Clinically, AEC syndrome differs from EEC syndrome by the near absence of digit malformations and the occurrence of eyelid fusions (ankyloblepharon) and severe skin erosions (1). Similarly, mutations in the gene encoding the transcription factor interferon regulatory factor 6 (IRF6) cause van der Woude syndrome (VWS) and popliteal pterygium syndrome (PPS), both of which are characterized by many ectodermal anomalies, including CL/CP (2). p63 and IRF6 mutations also have been implicated in nonsyndromic forms of CL/CP (i.e., forms of CL/CP in which the lip and/or palate are the only affected tissues) (3).

A first glimpse of the physiological roles of p63 and IRF6 came from studies of mutant mice. p63-null mice lack a stratified epidermis, teeth, hair, and several exocrine glands and display limb truncations as well as CL and CP $(4,5)$. The skin defects are caused by impaired proliferation and differentiation of epidermal keratinocytes (4). Mice either null for Irf6 (6) or homozygous for $R 84 C$ (7), a common IRF6 mutation in individuals with PPS, replicate several anomalies found in patients with VWS and PPS, including skin impairment and the aberrant intraoral epithelial adhesions that lead to CP. The abnormal development of the skin is due to the inability of epidermal keratinocytes to stop proliferating and to undergo terminal differentiation $(6,7)$.

The discovery that mutations in the genes encoding p63 and IRF6 cause human ectodermal dysplasia syndromes triggered a chain reaction of research aimed at unravelling their functions and identifying their targets during normal and abnormal epithelial development (4-8). Yet, their mode of action, regulation, and targets during normal lip and palate development and the mechanisms behind the genesis of CL/CP in both humans and mouse models are still largely unknown. However, in this issue of the JCI, Moretti et al. (9) and Thomason et al. (10) identify an intriguing relationship between p63 and IRF6 during palate development (well illustrated in Figure 5 in ref. 9) by using an arsenal of experimental strategies.

\section{IRF6 is a direct target of p63}

Studies of the functions of $\mathrm{p} 63$ are complicated by the existence of at least six different isoforms. Two separate transcription start sites generate the $\Delta \mathrm{Np} 63$ isoforms and the p53-like TAp63 isoforms. Transcripts of both the TAp 63 and $\Delta \mathrm{Np} 63$ isoforms undergo alternative splicing, generating three subtypes each, designated $\alpha, \beta$, and $\gamma(4)$. Here, the term $p 63$ is adopted to refer to experiments and data that do not dis- 

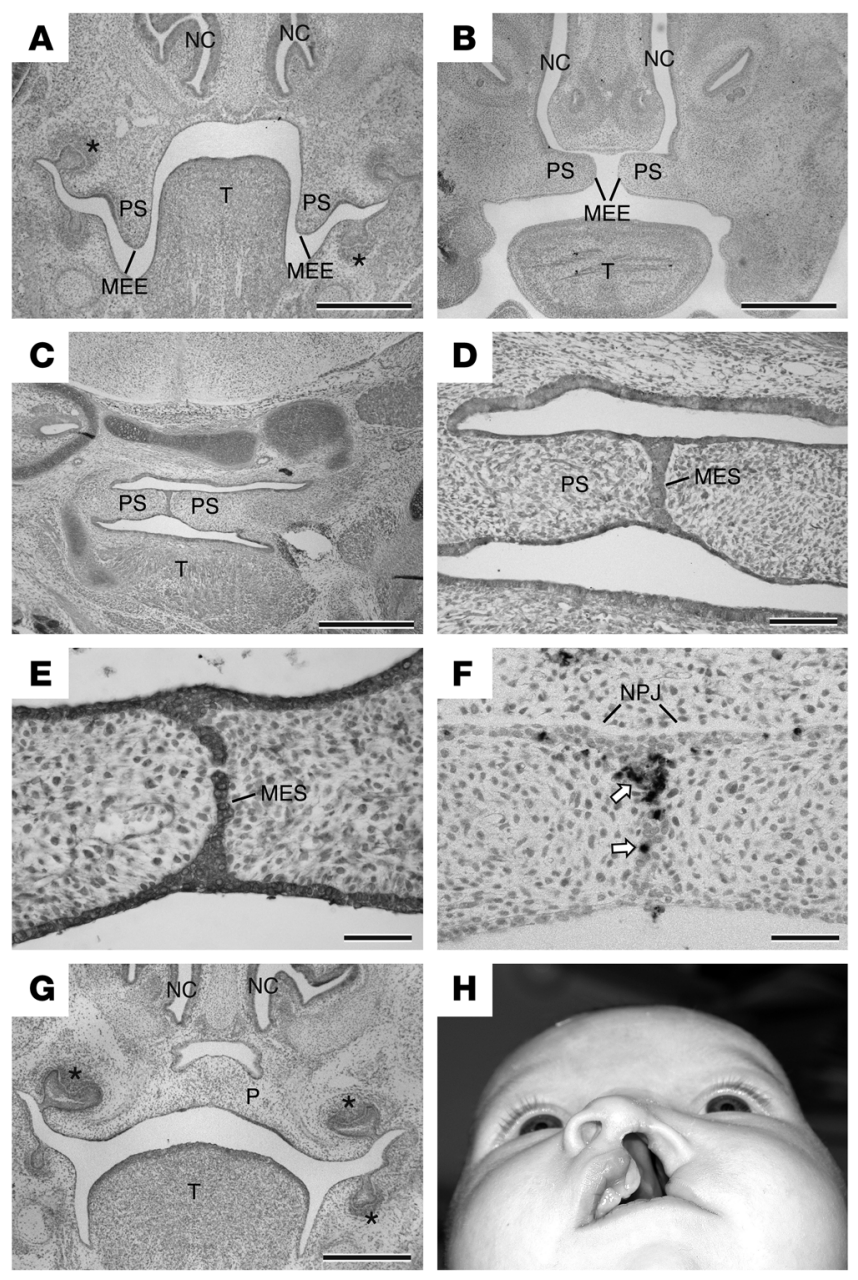

tinguish between the TAp63 and $\Delta \mathrm{Np} 63$ isoforms, while $\Delta \mathrm{Np} 63$ is used to describe experiments and findings that do not distinguish between the $\alpha, \beta$, and $\gamma$ subtypes of this isoform.

In the search for putative targets of p63, Moretti et al. (9) identified Irf6 as a positively regulated target of $\triangle \mathrm{Np} 63$. Given that IRF6 and $p 63$ are expressed in stratified epithelia, and because of the similarities between the organs affected in humans and mice with mutations in the genes encoding IRF6 and p63, Thomason and coworkers (10) hypothesized that these transcription factors may in fact interact. They generated compound heterozygotes for $p 63$ and $\operatorname{Irf6} 6^{R 84 C}\left(p 63^{+/-}\right.$ $\operatorname{Irf} 6^{+/ R 84 C}$ mice) and found that, in contrast to the single heterozygotes, which were devoid of CP, the double heterozygotes exhibited a fully penetrant $\mathrm{CP}$, indicating a genetic interaction between Irf6 and $p 63$.

Expression analyses using palates and epidermis from mice with mutations in p63 and keratinocytes and epidermis from patients with EEC and AEC syndromes, combined with in vitro mutagenesis, demonstrated that expression of IRF6 requires normal function of $\Delta \mathrm{Np} 63(9,10)$. Using in vitro genetic manipulation and biochemical assays, the authors show that IRF6 is directly activated by $\Delta \mathrm{Np} 63 \alpha(9,10)$ but not by TAp63 (9). Importantly, diseasecausing mutations in $p 63$ abrogated the ability of p63 to activate IRF6 transcription in vivo and in vitro $(9,10)$.

These findings are clinically important, as they suggest that IRF6 can acts as a modifier gene to affect $p 63$ disease outcomes and that polymorphisms in the newly identified p63-binding site, in an upstream enhancer element of IRF6 (10), may be involved in the subset of individuals with VWS and PPS, in which the causative IRF6 mutations have remained elusive, as well as in some forms of nonsyndromic orofacial clefting. As the p63-binding site upstream of the IRF6 gene was found to overlap with a recently identified enhancer element of IRF6, in which a single nucleotide polymorphism associated with cleft lip disrupts binding of the tran-

\section{Figure 1}

Development of the mouse secondary palate and human CL/CP. (A-G) Histological sections of mouse embryo heads, showing the development sequences of the secondary palate. (A) At E14, the PSs are vertically oriented in the oral cavity. (B) At E14.5, the PSs have elevated to a horizontal position above the dorsum of the tongue (T). (C and D) At E15, adhesion of the PSs, through their MEE, creates the MES. (D) A high-magnification image of the PS shown in C. (E) Immunohistochemistry showing expression of zonula occludens 1, a tight junction component (dark color), in the regressing MES and the rest of the palate epithelium. (F) Immunostaining for cleaved lamin A, showing apoptotic cells (dark spots indicated by arrows) in the MES and in the epithelial seam at the nasopalatine junction (NPJ), the site of adhesion between the PS and the nasal septum. (G) Successful closure of the palate $(P)$ at E15.5 leads to the separation of the oral and the nasal cavities (NC). (H) Infant with unilateral total clefts of the lip, alveolar ridge, primary palate, and secondary palate. Asterisks indicate tooth primordia. Scale bars: $500 \mu \mathrm{m}(\mathbf{A}-\mathbf{C}$ and $\mathbf{G})$; $100 \mu \mathrm{m}$ (D and F); and $50 \mu \mathrm{m}$ (E). scription factor AP-2 $\alpha$ (11), Thomason et al. (10) suggest it is likely that p63 and AP- $2 \alpha$ co-operate in the regulation of IRF6.

\section{Development of the secondary palate} The sequences of secondary palate development (i.e., development of the structure that separates the nasal cavity from the oral cavity, allowing simultaneous breathing and eating) in humans and mice are similar and tightly regulated by epithelial-mesenchymal interactions (3). Initially, the bilateral palatal shelves (PSs), which are composed of a core of mesenchyme covered by an epithelium, grow downward from the maxillary primordium (Figure 1A). Subsequently, the PSs elevate into a horizontal position (Figure 1B) and contact each other through their medial edge epithelia (MEE), which, after adhesion, form a transient medial epithelial seam (MES) made of nonproliferating cells (Figure 1, C and D). The periderm, a layer of flat cells that cover external epithelia in embryos, is required for establishing the first firm contact of MEE through filopodia 
(polarized cell protrusions). Adhesion of the opposing MEE occurs by junctional complex proteins (Figure 1E). Thereafter, apoptosis seals the fate of the MES (Figure 1F). The quick degeneration of the MES allows mesenchymal continuity and successful palate closure (Figure 1G). Similarly, upper lip and primary palate development proceed by growth, morphogenesis, and fusion of facial primordia (3). The primary palate forms the anterior-most structure of the upper jaw, the premaxilla, from which the four upper incisor teeth arise. Disruption of these steps results in CL/CP (Figure 1H). These sequences may seem simple; however, each stage of lip and palate development is governed by an impressive number of molecular pathways (3).

\section{Abnormal epithelial differentiation generates cleft palate}

Although the palatal MEE of $p 63^{+/-} \operatorname{Irf6} 6^{+/ R 84 C}$ compound heterozygote embryos were able to abut, they ignored each other, adhesion failed, and the palate remained cleft (10). In these mutants, an abnormal differentiation of the MEE and failure of polarization of peridermal cells prevented adhesion of the PS. In Irf6 ${ }^{R 84 C / R 84 C}$ embryos, the MEE fails to disintegrate at sites of ectopic adhesion, suggesting a defective epithelial differentiation (12).

In mice, abrogation of TGF- $\beta$ signaling in the MEE leads to a complete loss of Irf6 expression (3), suggesting that Irf6 functions downstream of TGF- $\beta$ during palatal fusion. Normal TGF- $\beta$ activity is an absolute requirement for MES disintegration by apoptosis, a prerequisite for fusion. Remarkably, loss of signaling via the type II TGF- $\beta$ receptor leads to $\mathrm{CP}$, as a result of unchecked proliferation of MES cells that fail to die (3, 13 ), a situation that seems to result from failure of induction of the TGF- $\beta$ target gene $p 21$, which encodes a cyclin-dependent kinase inhibitor (13). Interestingly, $\Delta \mathrm{Np} 63 \alpha$ has been shown to directly repress transcription of $p 21$ and the stratifin gene ( $S f n$ ) encoding another antiproliferative cell cycle mediator, and this activity is strongly diminished in cells expressing mutant forms of $\Delta \mathrm{Np} 63 \alpha(14)$. In mice, $S f n$ and Irf6 have been found to interact genetically (7).

All these observations point to intricate interactions between TGF- $\beta$ signaling, IRF6, and p63 during palatogenesis. TGF- $\beta$ signaling exerts roles in tumor suppression and metastasis, and recent findings point to a tumor suppressor function for $\mathrm{p} 63$, which promotes the oncogenic function of TGF- $\beta$ in cancer cells when it is lost subsequent to its TGF- $\beta$-dependent sequestration by mutant p53 (15). IRF6 mRNA and protein are totally lost in metastatic breast cancer cell lines and in invasive breast ductal carcinomas (8). These observations suggest that TGF- $\beta$, p63, and IRF6 also interact in cancer.

\section{IRF6 targets p63 to proteasomal degradation}

Inasmuch as $p 21$ expression is required for MES disintegration (13), and because $\Delta \mathrm{Np} 63 \alpha$ represses $p 21$ expression (14), one would imagine that for $p 21$ to be expressed in the MES, p63 must disappear. Indeed, Thomason et al. (10) found that in WT embryos, upon formation of the MES following adhesion of the MEE, p63 protein was spirited away, whereas Irf6 reached the pinnacle of its expression. Intriguingly, the MEE of Irf6 ${ }^{R 84 C / R 84 C}$ mutants remained p63 positive, indicating that p63 downregulation necessitates normal IRF6 function (10). Similarly, Moretti et al. (9) found a reciprocal relationship between IRF6 and p63 proteins, both in human skin sections and in differentiating keratinocytes, leading the authors to posit that following transcriptional activation of IRF6 by $\Delta \mathrm{Np} 63$, IRF6 promotes the decay of $\Delta \mathrm{Np} 63$, allowing keratinocytes to exit the cell cycle and differentiate. This hypothesis was validated by experimental assays showing that differentiation-induced decrease of $\Delta \mathrm{Np} 63$ protein levels and terminal differentiation of keratinocytes required IRF6 activity (9).

What happens to p63? Its fate turns out to be proteasomal degradation. While this finding concurs with those of previous studies, indicating proteasomal degradation of WT but not mutant $\Delta \mathrm{Np} 63$ (16-18), the treasure that was unearthed by Moretti et al. (9) was that IRF6 itself targets the $\Delta \mathrm{Np} 63 \alpha$ and $\Delta \mathrm{Np} 63 \beta$ isoforms to proteasomal degradation. Moreover, they show that a mutant $\Delta \mathrm{Np} 63$ protein corresponding to one that causes EEC syndrome is resistant to IRF6-mediated downregulation, whereas the product of $I R F 6^{R 84 C}$ is unable to induce $\Delta \mathrm{Np} 63$ protein downregulation. These elegant studies provide a clear explanation as to the perdurance of $\Delta \mathrm{Np} 63$ protein in the MEE of Irf6 $6^{R 84 C / R 84 C}$ mutant mice (10). Thus, WT IRF6 turns against its inducer; however, this treacherous act is intended for a good cause: preventing orofacial clefting and other ectodermal anomalies.

Together, the studies by Moretti et al. (9) and Thomason et al. (10) demonstrate that IRF6 and p63 function in a biological regu- latory loop to coordinate epithelial proliferation and differentiation. Furthermore, they show that disease-causing mutations in IRF6 or p63 interrupt this loop and lead to disruption in epithelial development and subsequently to severe malformations, including CL/CP.

While the findings of Moretti et al. (9) and Thomason et al. (10), together with the initial study of the mechanisms leading to CL in $p 63^{-/-}$embryos (5), enhance our understanding of the molecular and cellular events controlled by IRF6 and p63 during normal and abnormal development of the lip and palate, they clearly set the stage for human genetic studies to define the etiology of nonsyndromic forms of orofacial clefting. Recent advances (19) provide hope that direct targets of IRF6 can be identified and then assessed as potential causal genes in orofacial clefting. Since p63 and IRF6 are known to be involved in cancer $(1,8,15)$ and in view of the present findings $(9,10)$, it wouldn't be surprising if the p63IRF6 relationship were disrupted in cancer, albeit with variation inherent to neoplasia.

\section{Perspectives}

Naturally, the findings of Moretti et al. (9) and Thomason et al. (10) generate questions. How does IRF6 promote $\Delta \mathrm{Np} 63 \alpha$ proteasomal degradation? At least in cancer cells, stratifin seems to indirectly target phosphorylated $\Delta \mathrm{Np} 63 \alpha$ to the proteasome by escorting it out of the nucleus, while the proteasomal targeting itself is executed by RACK1, which has been suggested to function as an E3 ubiquitin ligase (20). However, there are precedents for SUMO1- and ubiquitinmediated proteasomal degradation of $\Delta \mathrm{Np} 63 \alpha(16-18)$. Is IRF6 engaged in direct or indirect induction of some key factor(s) in the sumoylation or ubiquitylation cascades? For example, SUMO1 itself or a specific ubiquitin ligase could be potential targets. Of note, several key players in lip and palate development have been shown to be substrates for sumoylation by SUMO1, and altered function of SUMO1 in humans and mice results in orofacial clefting (3). Compound heterozygous Sumo1 ${ }^{+/-} p 63^{+/-}$mice may prove informative in this regard. IRF6 is also a substrate for proteasomal degradation, following phosphorylation and ubiquitination (8); however, the molecular mechanisms behind its posttranslational modification are still unknown. How does the IRF6-p63 regulatory loop integrate into other signaling pathways under normal and diseased states? 
Collaboration between clinicians, geneticists, and basic scientists as well as the clever use of mouse models will certainly help address some of these questions and enhance our understanding of IRF6- and p63-related diseases, the ultimate beneficiaries being the afflicted patients and their families.

\section{Acknowledgments}

I am grateful to Anders Linde for preparing the figures and to Sara Rizell for providing the clinical photo. The author is supported by the Swedish Research Council-Medicine (grants 15181 and 20614), the Institute of Odontology at Sahlgrenska Academy, and the Thuréus Foundation.

Address correspondence to: Amel GritliLinde, Department of Oral Biochemistry, Sahlgrenska Academy, University of Gothenburg, Medicinaregatan 12F, SE-41390 Göteborg, Sweden. Phone: 46.31.786.3386; Fax: 46.31.418122; E-mail: amel@odontologi.gu.se.

\footnotetext{
1. Rinne T, Bruner HG, van Bokhoven H. P63-associated disorders. Cell Cycle. 2007;6(3):262-268.
}

2. Kondo S, et al. Mutations in IRF6 cause van der Woude and popliteal pterygium syndromes. Nat Genet. 2002;32(2):285-289.

3. Gritli-Linde A. The etiopathogenesis of cleft lip and cleft palate: Usefulness and caveats of mouse models. Curr Top Dev Biol. 2008;84:37-138.

4. Mikkola ML. P63 in skin appendage development. Cell Cycle. 2007;6(3):285-290.

5. Thomason HA, Dixon MJ, Dixon J. Facial clefting in Tp63 deficient mice result from altered Bmp4, Fgf8 and Shh signaling. Dev Biol. 2008;321(1):273-282.

6. Ingraham CR, et al. Abnormal skin, limb and craniofacial morphogenesis in mice deficient for interferon regulatory factor 6 (Irf6). Nat Genet. 2006;38(11):1335-1340.

7. Richardson RJ, et al. Irf6 is a key determinant of the keratinocyte proliferation-differentiation switch. Nat Genet. 2006;38(11):1329-1334

8. Bailey CM, Hendrix MJC. IRF6 in development and disease: A mediator of quiescence and differentiation. Cell Cycle. 2008;7(13):1925-1930.

9. Moretti F, et al. A regulatory feedback loop involving $\mathrm{p} 63$ and IRF 6 links the pathogenesis of 2 genetically different human ectodermal dysplasias. J Clin Invest. 2010;120(5):1570-1577.

10. Thomason HA, et al. Cooperation between the transcription factors p 63 and IRF6 is essential to prevent cleft palate in mice. J Clin Invest. 2010;120(5):1561-1569.

11. Rahimov F, et al. Disruption of an AP- $2 \alpha$ binding site in an IRF6 enhancer is strongly associated with cleft lip. Nat Genet. 2008;40(11):1341-1347.

12. Richardson RJ, Dixon J, Jiang R, Dixon MJ. Integration of IRF6 and Jagged 2 signalling is essential for controlling palatal adhesion and fusion competence. Hum Mol Genet. 2009;18(14):2632-2642.

13. Xu X, et al. Ectodermal Smad4 and p38 MAPK are functionally redundant in mediating TGF- $\beta /$ BMP signaling during tooth and palate development. Dev Cell. 2008;15(2):322-329.

14. Westfall MD, Mays DJ, Sniezek JC, Pietenpol JA. The $\Delta \mathrm{Np} 63 \alpha$ phosphoprotein binds the $\mathrm{p} 21$ and $14-3-3 \sigma$ promoters in vivo and has transcriptional repressor activity that is reduced by Hay-Wells syndrome-derived mutations. Mol Cell Biol. 2003;23(7):2264-2276.

15. Adorno $M$, et al. A mutant-p53/Smad complex opposes p 63 to empower Tgf $\beta$-induced metastasis. Cell. 2009;137(1):87-98.

16. Ghioni $\mathrm{P}$, et al. The protein stability and transcriptional activity of $\mathrm{p} 63 \alpha$ are regulated by SUMO-1 conjugation. Cell Cycle. 2005;4(1):183-190.

17. Rossi M, et al. The E3 ubiquitin ligase Itch controls the protein stability of p63. Proc Natl Acad Sci US A. 2006;103(34):12753-12758

18. Li Y, Zhou Z, Chen C. WW domain-containing E3 ubiquitin protein ligase 1 targets $\mathrm{p} 63$ transcription factor for ubiquitin-mediated proteasomal degradation and regulates apoptosis. Cell Death Differ. 2008;15(12):1941-1951.

19. Little HJ, et al. Missense mutations that cause van der Woude syndrome and popliteal pterygium syndrome affect the DNA-binding and transcriptional activation functions of IRF6. Hum Mol Genet. 2009;18(3):535-545.

20. Fomenkov A, et al. RACK1 and Stratifin target $\Delta \mathrm{Np} 63 \alpha$ for a proteasome degradation in head and neck squamous cell carcinoma cells upon DNA damage. Cell Cycle. 2004;3(10):1285-1295.

\section{Cryptococci at the brain gate: break and enter or use a Trojan horse?}

Arturo Casadevall

Department of Microbiology and Immunology and Medicine, Albert Einstein College of Medicine, New York, New York, USA.

\begin{abstract}
The mechanism by which Cryptococcus neoformans invades the central nervous system is fundamental for understanding pathogenesis because cryptococcosis commonly presents as meningoencephalitis. There is evidence for both direct invasion of the endothelial cells lining the brain vasculature and a "Trojan horse" mechanism whereby cryptococci enter the central nervous system after macrophage ingestion. However, in this issue of the JCI, Shi et al. use intravital microscopy to reveal that brain invasion by C. neoformans follows a capillary microembolic event. They find that after suddenly stopping in brain capillaries, cryptococci cross into the central nervous system in a process that is urease dependent, requires viability, and involves cellular deformation. This observation provides evidence for direct brain invasion by C. neoformans, but a consideration of all the currently available evidence suggests a role for both direct and phagocyte-associated invasion. Hence, the remarkable neurotropism of $C$. neoformans may have more than one mechanism.
\end{abstract}

Cryptococcus neoformans is a soil-dwelling fungus that emerged in the late twentieth century as a major human pathogen

Conflict of interest: Arturo Casadevall is a consultant and holds stock options in Pain Therapeutics Inc.

Citation for this article: J Clin Invest. 2010;

120(5):1389-1392. doi:10.1172/JCI42949. because of its propensity to cause lethal meningoencephalitis. The burden of cryptococcosis is estimated to approach one million cases per year, with a mortality that exceeds that from tuberculosis (1). C. neoformans is acquired by inhalation of dehydrated cells or spores (2). Serologic surveys indicate a high prevalence of human infection, which is likely to be first acquired in childhood (3). Although infection is common, disease is rare, and cryptococcosis occurs primarily in hosts with impaired immunity, such as patients with AIDS, organ transplant recipients, and those treated with immunosuppressive therapies (2). Hence, normal immune responses are believed to control infection in the lung. Extrapulmonary dissemination is therefore invariably associated with disease, with meningoencephalitis being the most common clinical presentation of cryptococcosis. To cause meningoencephalitis, C. neoformans must cross several epithelial and/or endothelial cell layers, first to leave the lung and then to reach the brain. How does a soil-dwelling organism that has no need for animal pathogenesis for survival such as C. neoformans reach the brain to cause meningoencephalitis? In this issue of the JCI, Shi et al. (4) shed new light on this subject by applying 